\title{
Inhibition of ACE Retards Tau Hyperphosphorylation and Signs of Neuronal Degeneration in Aged Rats Subjected to Chronic Mild Stress
}

\author{
Said AbdAlla, ${ }^{1}$ Ahmed el Hakim, ${ }^{2}$ Ahmed Abdelbaset, ${ }^{3}$ \\ Yasser Elfaramawy, ${ }^{3}$ and Ursula Quitterer ${ }^{1,4}$ \\ ${ }^{1}$ Molecular Pharmacology Unit, Department of Chemistry and Applied Biosciences, ETH Zurich, \\ Winterthurerstrasse 190, 8057 Zurich, Switzerland \\ ${ }^{2}$ International Neuroscience Institute (INI) Hannover, Rudolf-Pichlmayr-Strasse 4, 30625 Hannover, Germany \\ ${ }^{3}$ Medical Research Centre (MRC), Ain Shams University Hospitals, Abassia, Cairo 11591, Egypt \\ ${ }^{4}$ Institute of Pharmacology and Toxicology, Department of Medicine, University of Zurich, Winterthurerstrasse 190, \\ 8057 Zurich, Switzerland
}

Correspondence should be addressed to Said AbdAlla; said.abdalla@pharma.ethz.ch

Received 20 August 2015; Accepted 12 November 2015

Academic Editor: Grazia D’Onofrio

Copyright (C) 2015 Said AbdAlla et al. This is an open access article distributed under the Creative Commons Attribution License, which permits unrestricted use, distribution, and reproduction in any medium, provided the original work is properly cited.

\begin{abstract}
With increasing life expectancy, Alzheimer's disease (AD) and other types of age-associated dementia are on the rise worldwide. Treatment approaches for dementia are insufficient and novel therapies are not readily available. In this context repurposing of established drugs appears attractive. A well-established class of cardiovascular drugs, which targets the angiotensin II system, is such a candidate, which currently undergoes a paradigm shift with regard to the potential benefit for treatment of neurodegenerative symptoms. In search for additional evidence, we subjected aged rats to chronic unpredictable mild stress, which is known to enhance the development of $\mathrm{AD}$-related neuropathological features. We report here that four weeks of chronic mild stress induced a strong upregulation of the hippocampal angiotensin-converting enzyme (Ace) at gene expression and protein level. Concomitantly, tau protein hyperphosphorylation developed. Signs of neurodegeneration were detected by the significant downregulation of neuronal structure proteins such as microtubule-associated protein 2 (Map2) and synuclein-gamma $(S n c g)$. Ace was involved in neurodegenerative symptoms because treatment with the brain-penetrating ACE inhibitor, captopril, retarded tau hyperphosphorylation and signs of neurodegeneration. Moreover, $A C E$ inhibitor treatment could counteract glutamate neurotoxicity by preventing the downregulation of glutamate decarboxylase 2 (Gad2). Taken together, $A C E$ inhibition targets neurodegeneration triggered by environmental stress.
\end{abstract}

\section{Introduction}

Alzheimer's disease (AD) is the most common form of dementia. While genetic factors are causally linked to familial $\mathrm{AD}$, the pathogenesis of the predominant late-onset sporadic $\mathrm{AD}$ is diverse and less defined. Age is one of the bestdocumented risk factors for sporadic $\mathrm{AD}$, which acts in concert with a wide array of brain insult-promoting vascular and metabolic factors such as hypertension, ischemia, diabetes, high cholesterol, and different forms of environmental stress and stress-related psychiatric symptoms, that is, depression and anxiety [1-3].

Experimental models of $\mathrm{AD}$ often reproduce genetic alterations whereas the impact of additional brain-damaging factors is more difficult to assess. The chronic unpredictable mild stress (CUMS) model imitates psychiatric risk factors such as psychological, psychosocial, and physical stress [4]. In agreement with the well-established impact of stress on neuronal viability, the model promotes signs of neuronal degeneration such as decreased synaptic transmission at 
hippocampal CA1-CA3 synapses, impaired neurogenesis, and cognitive dysfunction [5-7]. The sensitivity to stress in this model increases with age, a major factor of $\mathrm{AD}$ pathogenesis [8]. The relationship to AD is further supported by several studies, which show that the CUMS procedure worsens disease progression in genetic $\mathrm{AD}$ mouse models [9-11].

Moreover, the chronic mild stress protocol induces major neuropathological hallmarks of $\mathrm{AD}$, that is, enhanced $\beta$ amyloid $(\mathrm{A} \beta)$ generation, tau hyperphosphorylation, and the appearance of various other AD markers $[9,11,12]$. In this respect, the chronic mild stress model could complement genetic AD models because the CUMS model solely relies on endogenously expressed proteins. In view of the recent failure of large clinical trials, which targeted $\beta$-amyloid plaques $[13,14]$, such alternative models, which imitate the disease process before the accumulation of $\mathrm{A} \beta$ plaques, could become of substantial value.

Inefficient $\mathrm{A} \beta$ plaque-targeting approaches also raise the necessity to identify players in AD pathogenesis, which could interrupt the disease process at an earlier stage [15]. For one player in $\mathrm{AD}$ pathogenesis a paradigm shift is currently on the way, which is the angiotensin system, notably the angiotensin-converting enzyme $(A C E)$. While many studies considered $A C E$ primarily as an enzyme that could promote proteolysis of $\beta$-amyloid in vitro [16-18], recent experimental and clinical studies provide strong evidence that inhibition of $A C E$-dependent angiotensin II generation in vivo could actually reduce signs of neurodegeneration in experimental AD models $[19,20]$ and slow the cognitive decline of patients with Alzheimer's disease [21-25].

In view of this emerging development, we aimed to further analyse the impact of ACE inhibition on early signs of neurodegeneration and applied the chronic unpredictable mild stress model in our study.

\section{Materials and Methods}

2.1. Chronic Unpredictable Mild Stress Model. Male Wistar rats (15 months of age) were subjected to the chronic unpredictable mild stress (CUMS) battery for four weeks. The CUMS protocol was performed essentially as described previously $[8,9]$. After four weeks of stress, more than $90 \%$ of stressed rats showed signs of anhedonia as documented by a decrease in sucrose preference compared to nonstressed controls (i.e., $<50 \%$ of sucrose consumption compared to nonstressed controls). The age-matched control group was housed under standard conditions and had free access to food and water. The captopril treatment group received captopril in drinking water $(50 \mathrm{mg} / \mathrm{kg} / \mathrm{day}$, dissolved fresh every day) during the CUMS protocol. After 28 days of stress, at an age of 16 months, $4 \mathrm{~h}$ before the beginning of the dark phase, rats were anesthetized with ketamine and xylazine (100 mg/kg and $10 \mathrm{mg} / \mathrm{kg}$, i.p.), and brains were removed and processed for immunohistology $[9,20]$. For whole genome microarray gene expression profiling, biochemical analyses, and immunoblotting, the hippocampus was dissected out on ice and processed for further use as detailed below.
All animal experiments were performed in accordance with the NIH guidelines and were approved by the local committee on animal research (MRC, Ain Shams University Hospitals, Cairo, Egypt).

2.2. Whole Genome Microarray Gene Expression Profiling of Hippocampal Gene Expression. For whole genome microarray gene expression analysis, total hippocampal RNA was isolated from stressed rats and age-matched nonstressed control rats. Total RNA was processed for whole genome microarray gene expression profiling and hybridized to the GeneChip Rat Genome 2302.0 Array (more than 31000 probe sets, Affymetrix) as detailed previously [20]. The total RNA from 3 animals was pooled for one gene chip, and two gene chips are presented for each group. Microarrays were scanned with the Affymetrix GeneChip Scanner 7G, and signals were processed with a target value of 300 using GCOS (version 1.4, Affymetrix). Applied selection criteria for differently expressed genes (2-fold change requirement, just alpha, no false discovery correction, and $P<0.05)$ were validated specifically for drug treatment effects [20] and follow the guidelines of the Microarray Quality Control (MAQC) project for the identification of reproducible gene lists [26, 27]. Probe sets with significant difference $(P<0.05$ and $\geq 2$-fold difference, with call present and/or signal intensity $\geq 100$ ) between stressed rats and nonstressed, age-matched control rats were used for GO classification. Microarray data are available at the NCBI GEO repository (GSE72062).

Gene expression of Ace and Map2 in the hippocampus of stressed rats and nonstressed controls was determined by real-time quantitative (q) RT-PCR with a LightCycler 480 (Roche Diagnostics). Sequences of the forward and reverse primers were as follows: Ace forward $5^{\prime}$-GATTGCAGCCGGGCAACTTTTC-3'; Ace reverse $5^{\prime}$-CGGATCCGATGATCCTTCGC-3 $3^{\prime}$; Map 2 forward $5^{\prime}$-CACTGGAAGAAGCCTCGAAGA-3 ${ }^{\prime}$; Map2 reverse $5^{\prime}$-CACGGGCATTTCGATGAACC- $3^{\prime}$.

2.3. Immunoblotting, Immunohistology, and Biochemical Methods. Immunoblot detection of hippocampal proteins was performed with hippocampal tissue extracts as described $[9,20]$. For immunohistology and immunofluorescence analysis, we used paraffin-embedded brain sections obtained from stressed and nonstressed rats $(8 \mu \mathrm{m}$, taken at $50 \mu \mathrm{m}$ intervals, $10-15$ sections per set). The following antibodies were used for immunoblotting, immunofluorescence, and immunohistology: anti-Ace antibodies raised in rabbit against an antigen corresponding to amino acids 720-750 of mouse Ace [20]; anti-angiotensin II antibodies raised in rabbit against synthetic angiotensin II [20]; anti-APP/A $\beta$ antibodies raised in rabbit against a peptide corresponding to amino acids $672-714$ of human APP [20]; anti-AT1R antibodies raised in rabbit against an antigen corresponding to amino acids 306-359 of the mouse Agtrla sequence [20]; anti-Gad65 antibody (clone N-GAD65, developed in mouse against a synthetic peptide corresponding to amino acids 4-22 of human GAD65); anti-Grin3a antibodies raised in rabbit against a synthetic peptide derived from 


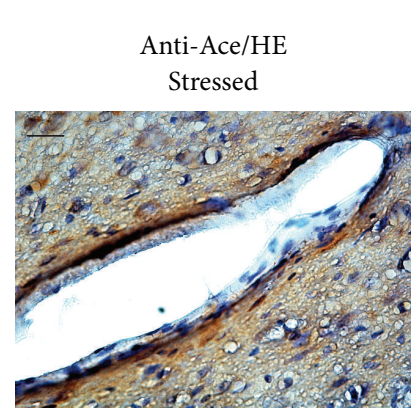

(a)

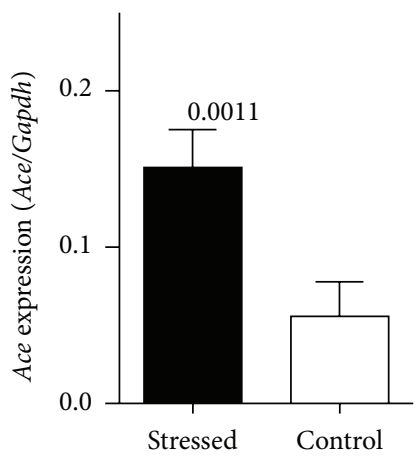

(d)

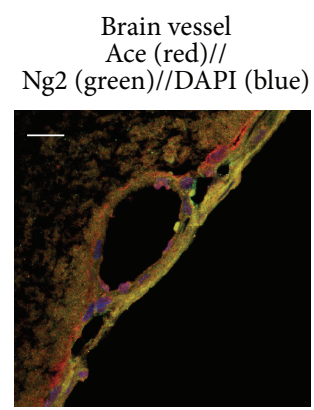

(b)

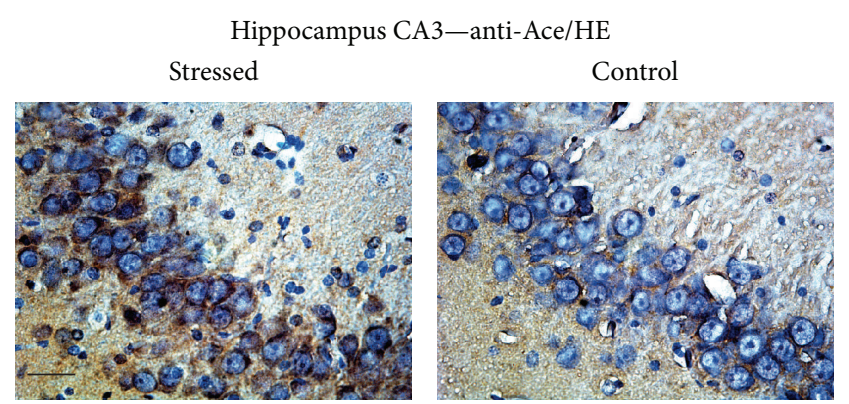

(c)

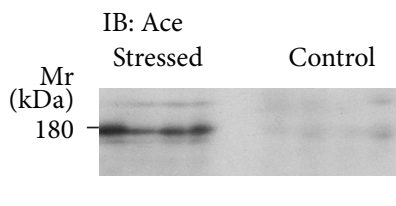

(e)

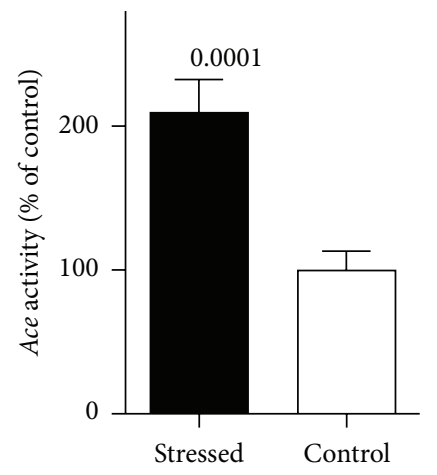

(f)

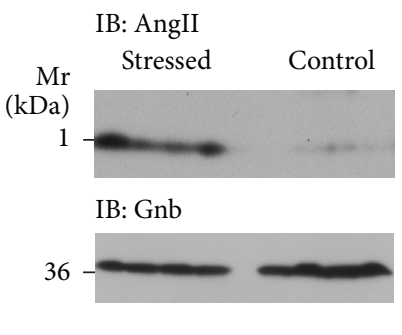

(g)

FIGURE 1: Chronic unpredictable mild stress induced upregulation of hippocampal Ace protein and gene expression. (a) Immunohistological localization of Ace in a brain vessel of a stressed rat. Nuclei were stained with hematoxylin (HE); bar: $20 \mu \mathrm{m}$. (b) Immunofluorescence localization of Ace (red) and the pericyte marker Ng2 (green) in a brain vessel of a stressed rat. Ace was detected with affinity-purified rabbit anti-Ace antibodies followed by $\mathrm{F}(\mathrm{ab})_{2}$ fragments of Alexa Fluor 546-labeled (red) secondary antibodies, and Ng2 was detected with affinity-purified murine anti-NG2 antibody followed by $\mathrm{F}(\mathrm{ab})_{2}$ fragments of Alexa Fluor 488-labeled (green) secondary antibodies. Nuclei were stained with DAPI (bar: $20 \mu \mathrm{m}$ ). (c) Immunohistological localization of Ace in hippocampal CA3 neurons of a stressed rat (left) relative to a nonstressed control (right). Nuclei were stained with hematoxylin (HE); bar: $20 \mu \mathrm{m}$. Histological experiments are representative of 4 rats/group (a-c). (d) Hippocampal Ace gene expression was determined by qRT-PCR and is presented as the ratio of Ace/Gapdh expression ( \pm s.d.; $n=4 ; P=0.0011$ ). (e) Immunoblot detection of the hippocampal Ace protein with anti-Ace antibodies in stressed rats relative to nonstressed controls ( $n=4$ /group). (f) Hippocampal Ace activity of stressed rats relative to nonstressed controls (i.e., $100 \%$; \pm s.d., $n=8$; $P=0.0001$ ). (g) The hippocampal angiotensin II content was determined by immunoblot in stressed rats relative to nonstressed controls (upper panel). The lower panel shows a control immunoblot, which detects Gnb ( $n=4$ /group).

the extracellular domain of rat Grin3a (Abcam); anti-MAP2 mouse monoclonal antibody generated against bovine microtubule-associated protein, MAP2 (Clone AP-20); antichondroitin sulfate proteoglycan NG2 mouse monoclonal antibody generated against a truncated form of NG2 (MAB5384); anti-PHF-tau pSer202 and pThr 205 antibody (mouse monoclonal antibody, clone AT8; Pierce); and antiSncg antibodies raised in goat against an internal region of human SNCG (Santa Cruz Biotechnology).

Hippocampal Ace activity was assessed with a fluorogenic substrate (Abz-FRK(Dnp)-P; Biomol) as described [20], and determination of hippocampal glutamic acid decarboxylase (Gad) enzyme activity was performed as detailed previously [8].

2.4. Statistical Analysis. The results are presented as means \pm s.d. We used unpaired two-tailed Student's $t$-test for comparisons between two groups. Statistical significance was set at a $P$ value of $<0.05$, unless otherwise specified.

\section{Results}

3.1. Chronic Unpredictable Mild Stress Induced Upregulation of Hippocampal Ace Protein and Gene Expression. Chronic stress worsens the progression of $\mathrm{AD}$ in patients and transgenic disease models [9-11] but underlying mechanisms are incompletely understood. Because the angiotensinconverting enzyme (ACE) is upregulated in brains of $\mathrm{AD}$ patients and mice [20], we asked for an interrelationship between Ace and stress. We used the chronic unpredictable mild stress model to investigate the role of Ace in stressinduced signs of neurodegeneration. Initially, we analysed the localization of Ace in stressed and nonstressed rat brain. Complementary to previous data with AD mice [20], endothelial cells lining a brain vessel of a stressed rat showed strong immunostaining for Ace (Figure 1(a)). Immunofluorescence localization of the pericyte marker, chondroitin sulfate proteoglycan $\mathrm{Ng}$, indicated that Ace was predominantly present in vascular endothelial cells whereas adjacent pericytes showed weaker staining for Ace (Figure 1(b)). 
We further investigated the localization of Ace by immunohistology and focused on the hippocampal area because chronic mild stress induces profound changes at hippocampal CA3-CA1 synapses [7], that is, the brain area, which is highly susceptible to $\mathrm{AD}$-related neuronal damage. In agreement with previous results [20], immunohistology analysis detected Ace immunoreactivity in cell bodies of hippocampal CA3 neurons (Figure 1(c)). Moreover, immunohistology revealed a strong increase in Ace immunoreactivity in hippocampal CA3 neurons of a stressed rat relative to that of a nonstressed control (Figure 1(c)).

In agreement with the increased Ace immunoreactivity, the gene expression level of hippocampal Ace was also significantly increased in stressed rats, that is, the hippocampal Ace gene expression level was $2.72 \pm 0.43$-fold higher in stressed rats compared to that in nonstressed controls $(P=0.0011$; Figure 1(d)). Concomitantly, with Ace gene expression, the increased hippocampal Ace protein level of stressed rats was detected in immunoblot (Figure 1(e)). The increase in Ace protein was accompanied by a significantly elevated hippocampal Ace activity; that is, the hippocampal Ace activity was $2.09 \pm 0.24$-fold higher $(P=0.0001)$ in stressed rats compared to that in nonstressed controls (Figure 1(f)). The elevated Ace activity was also reflected by an increased hippocampal angiotensin II content in stressed rats relative to nonstressed controls (Figure $1(\mathrm{~g})$ ). Taken together, chronic unpredictable mild stress led to a significant upregulation of the Ace protein in the hippocampal area of stressed rats. As a consequence of Ace upregulation, the hippocampal angiotensin II content in stressed rats was elevated compared to that in nonstressed controls.

\subsection{Tau Hyperphosphorylation in the Hippocampus of Stressed} Rats. The strong upregulation of Ace in the hippocampus of stressed rats prompted us to search for signs of hippocampal neurodegeneration because previous data showed a causal relationship between Ace upregulation and signs of neurodegeneration in a genetic model of $\mathrm{AD}$ [20]. In this context we determined the level of tau hyperphosphorylation as a hallmark of AD. Immunohistological analysis demonstrated that four weeks of chronic unpredictable mild stress induced a strong increase in the level of phosphorylated tau protein in the hippocampus of an aged rat, as detected in a hippocampal section with anti-PHF (AT8) antibody (Figure $2(\mathrm{a})$ ). Immunohistology also revealed that the increased tau phosphorylation was predominant in neurons of the CA1CA3 region (Figure 2(a)). In contrast, PHF antibody staining was negligible in hippocampal neurons of the nonstressed control (Figure 2(a)).

Immunoblot detection of phosphorylated tau in hippocampal tissue extracts confirmed the immunohistology data and showed an increased level of hyperphosphorylated tau protein in stressed rats compared to that in nonstressed controls (Figure 2(b)). As an additional control, the total level of hippocampal tau protein was not different between stressed and nonstressed rats (Figure 2(b), lower panel). Taken together, the upregulation of Ace and angiotensin II in hippocampal neurons of stressed rats was accompanied by tau hyperphosphorylation.
Ace-dependent angiotensin II generation promotes signs of hippocampal neurodegeneration by activation of the AT1 receptor (AT1R) in a genetic AD model [20]. Complementary to those data, immunofluorescence analysis of a stressed rat brain showed AT1R immunoreactivity in hippocampal CA1 neurons, which also displayed accumulation of hyperphosphorylated tau protein (Figure 2(c)). Concomitantly, neuronal loss of a highly PHF-positive CA1 neuron was detected (Figure 2(c)). Thus, stress promoted Ace-dependent release of the AT1R-stimulating angiotensin II peptide together with tau hyperphosphorylation in hippocampal AT1R-positive neurons.

\subsection{Whole Genome Microarray Gene Expression Profiling} Detected Stress-Induced Signs of Hippocampal Neurodegeneration. We further investigated the impact of stress on signs of neurodegeneration and performed whole genome microarray gene expression profiling of hippocampal gene expression. Gene ontology (GO) analysis searched for differently expressed neuron-specific genes by applying neuronspecific GO terms, that is, neuron, axon, dendrite, and synapse. Following established selection criteria for significantly different probe sets $(\geq 2$-fold difference and $P<$ $0.05)$, the GO analysis documented that stress triggered the significant downregulation of neuron-specific genes, which were categorized according to the cellular localization of the respective proteins, that is, cytoplasm and membrane (Figures 3(a) and 3(b)).

The list of significantly downregulated genes encompasses major neuronal structure proteins such as microtubuleassociated protein 2 (Map2) and synuclein-gamma, Sncg (Figure 3(a)). Because the loss of neuronal Map2 is a characteristic feature of $\mathrm{AD}$-related neurodegeneration, which is causally linked to stress-induced tau hyperphosphorylation $[9,28]$, we performed immunolocalization of the hippocampal Map2. Immunohistology analysis detected the decreased Map2 content in hippocampal CA1 neurons of a stressed rat compared to that of a nonstressed control (Figure 3(c)). The stress-induced downregulation of the hippocampal Map2 protein was further confirmed by qRT-PCR (Figure 3(d)). Together these data indicate that chronic unpredictable mild stress led to tau hyperphosphorylation and decreased the level of neuron-specific genes such as Map2. The decreased expression of neuronal structure proteins complements previous data, which show that chronic mild stress triggers alterations of hippocampal synapses and promotes a decrease in dendritic spine density [7].

The expression of glutamic acid decarboxylase 2 ( Gad65/ $\mathrm{Gad} 2$ ) was also downregulated by chronic unpredictable mild stress (Figure 3(a)). The decreased Gad65 expression could account for the significantly decreased hippocampal Gad enzyme activity, which was measured in hippocampal tissue extracts of stressed rats compared to that of nonstressed controls (Figure 3(e)). The ensuing decreased metabolism of the excitatory neurotransmitter, glutamate, could be partially responsible for the increased tau phosphorylation under chronic mild stress $[8,29]$. In addition to the decreased glutamate degradation, the expression of the inhibitory and 


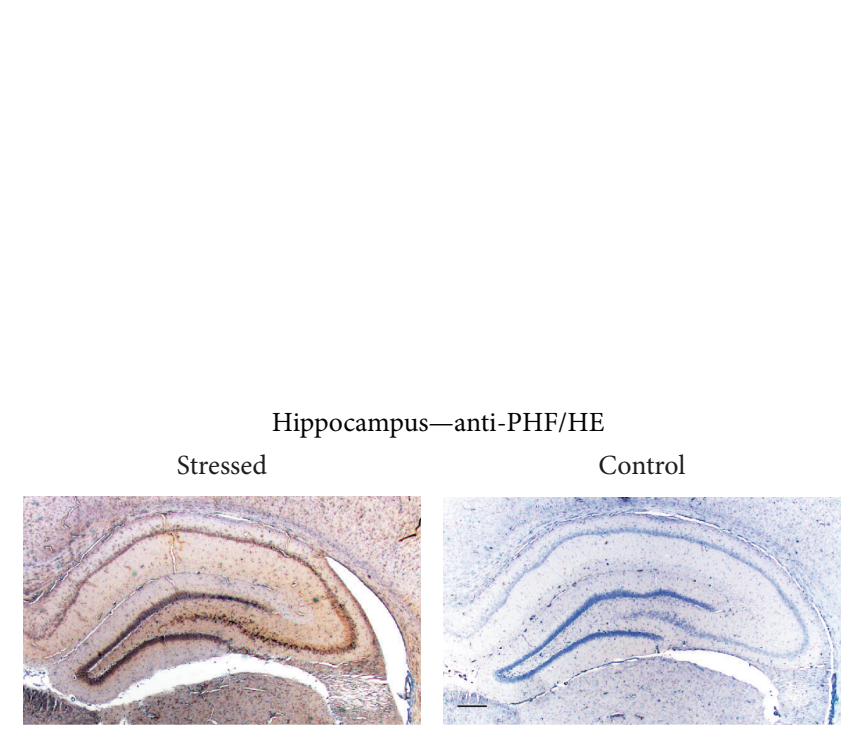

(a)

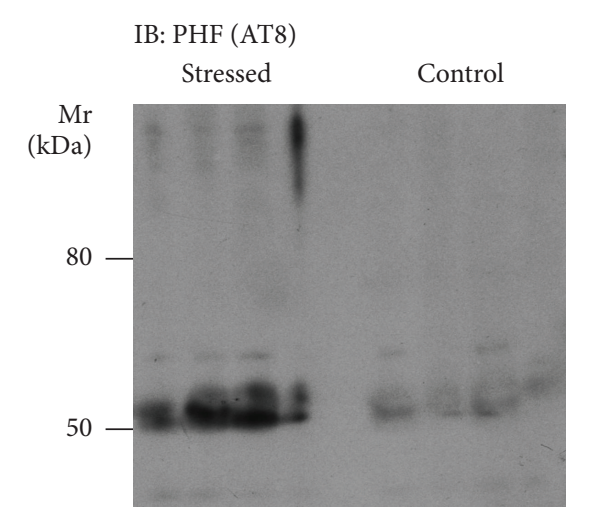

IB: tau (total)

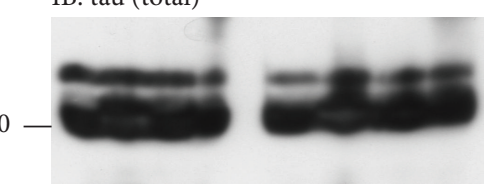

(b)

Hippocampus (stressed) AT1R (green)//PHF (red)
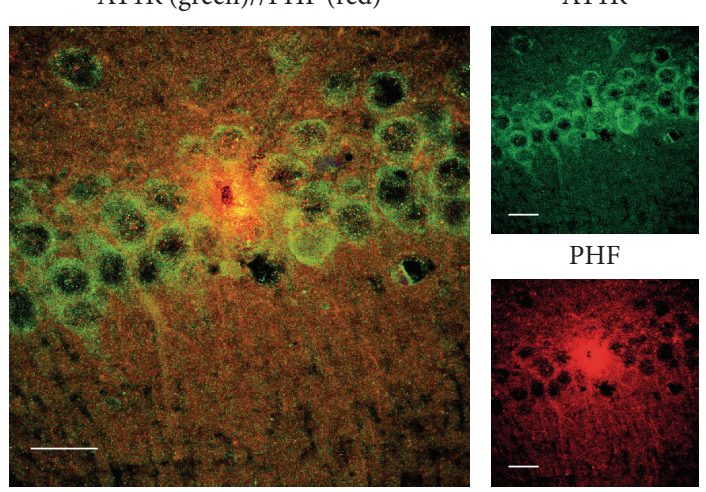

(c)

FIGURE 2: Tau hyperphosphorylation in the hippocampus of stressed rats. (a) Immunohistological detection of the hyperphosphorylated tau protein (anti-PHF) in the hippocampus of a stressed rat (left panel) relative to a nonstressed control (right panel). Nuclei were counterstained with hematoxylin (HE; bar: $200 \mu \mathrm{m}$ ). (b) Immunoblot detection of hyperphosphorylated tau in hippocampal extracts of stressed rats relative to nonstressed controls was performed with anti-PHF antibody (IB: PHF (AT8)). The lower panel is a control immunoblot detecting total tau protein $(n=4 /$ group). (c) Immunofluorescence localization of AT1R (green) and PHF (red) in hippocampal CA1 neurons of a stressed rat (bar: $20 \mu \mathrm{m})$. The AT1R was detected with affinity-purified rabbit anti-AT1R antibodies followed by F(ab) ${ }_{2}$ fragments of Alexa Fluor 488labeled (green) secondary antibodies, and hyperphosphorylated tau was visualized with affinity-purified mouse anti-PHF antibody followed by $\mathrm{F}(\mathrm{ab})_{2}$ fragments of Alexa Fluor 546-labeled secondary antibodies (red). Histological experiments are representative of 4 rats/group ((a) and $(c))$.

neuroprotective NMDA receptor subtype Grin3a (NR3A) $[30,31]$ was also downregulated by stress (Figure 3(b)). Immunohistology data complemented the microarray study and showed abundant membrane-localized Grin3a protein in hippocampal CA1 neurons of a nonstressed control whereas the neuronal Grin3a protein was barely detectable in the hippocampal neurons of a stressed rat (Figure 3(f)). As a consequence of stress-induced Grin3a (NR3A) downregulation, glutamate excitotoxicity, for example, triggered by decreased Gad enzyme activity, could be further augmented [30, 31]. Taken together, whole genome microarray gene expression profiling detected signs of neurodegeneration and markers of enhanced glutamate excitotoxicity in the hippocampus of stressed rats.

\subsection{Treatment with the Brain-Penetrating ACE Inhibitor,} Captopril, Retarded Signs of Neurodegeneration Induced by Chronic Mild Stress. In view of the strongly upregulated Ace protein, we asked whether treatment with the brainpenetrating ACE inhibitor, captopril, could affect the process of neurodegeneration triggered by stress. Aged rats were treated with captopril during the chronic unpredictable mild stress procedure. As a sign of neurodegeneration, we 


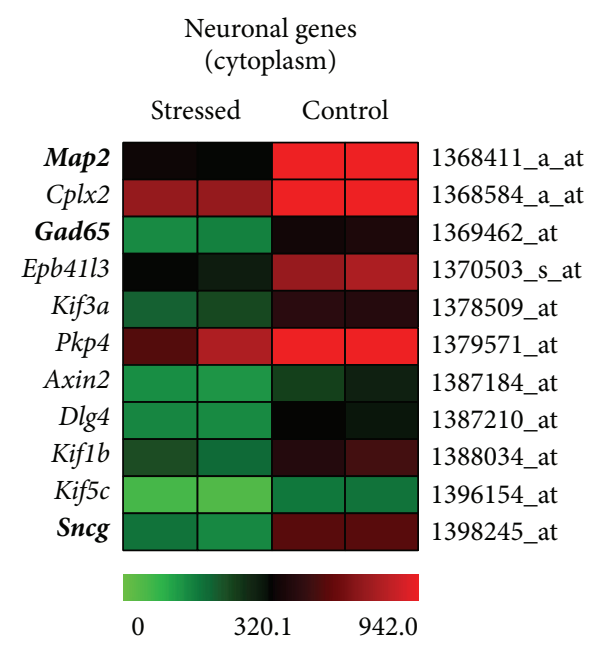

(a)

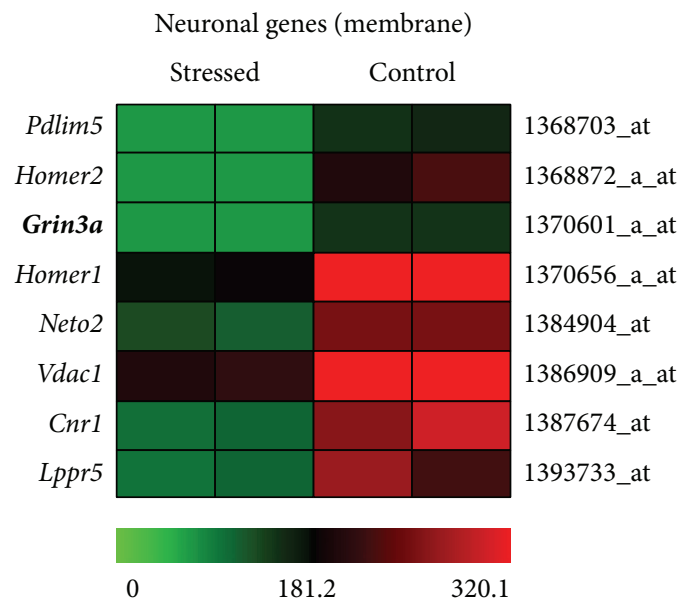

(b)

Hippocampus CA1—anti-Map2/HE

Stressed

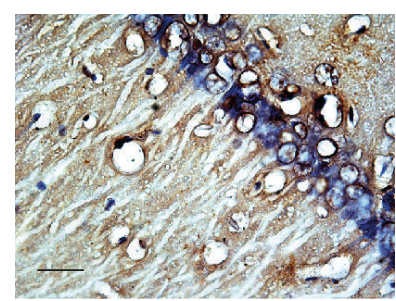

Control

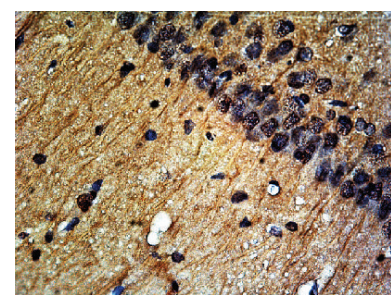

(c)

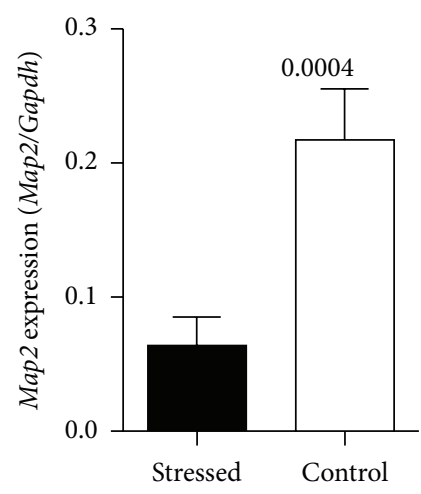

(d)

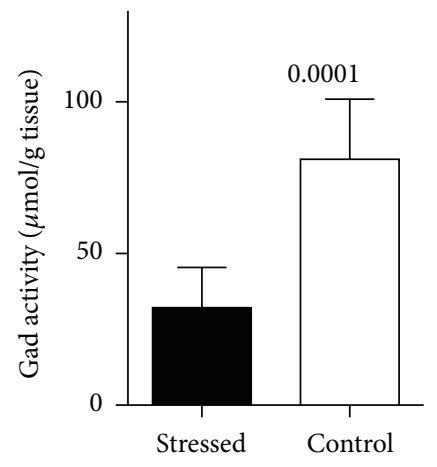

(e)

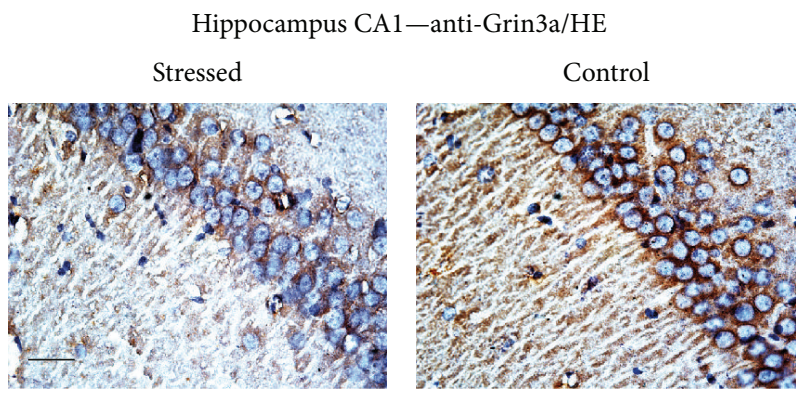

(f)

FIGURE 3: Whole genome microarray gene expression profiling detected stress-induced signs of hippocampal neurodegeneration. (a) and (b) Whole genome microarray gene expression profiling of hippocampal gene expression documents that stress promoted the significant downregulation of probe sets detecting neuron-specific genes with cytosolic localization (a) or membrane localization (b) according to GO analysis. All probe sets were significantly downregulated in the hippocampus of stressed rats relative to nonstressed controls $(P<0.05$ and $\geq 2$-fold difference). The heat map visualizes signal intensities (centered to the median value). (c) Immunohistological detection of Map2 in hippocampal CA1 neurons of a stressed rat (left panel) relative to a nonstressed control (right panel; bar: $20 \mu \mathrm{m}$ ). (d) Hippocampal Map2 expression level was determined by qRT-PCR and is presented as the ratio of Map2/Gapdh expression $( \pm$ s.d.; $n=4 ; P=0.0004)$. (e) Hippocampal Gad activity of stressed rats relative to nonstressed controls ( \pm s.d.; $n=8 ; P=0.0001$ ). (f) The decreased hippocampal Grin3a protein level of a stressed rat (left panel) relative to that of a nonstressed control (right panel) was detected by immunohistology in hippocampal CA1 neurons; bar: $20 \mu \mathrm{m}$. Immunohistology data are representative of 4 rats/group ((c) and (f)). 


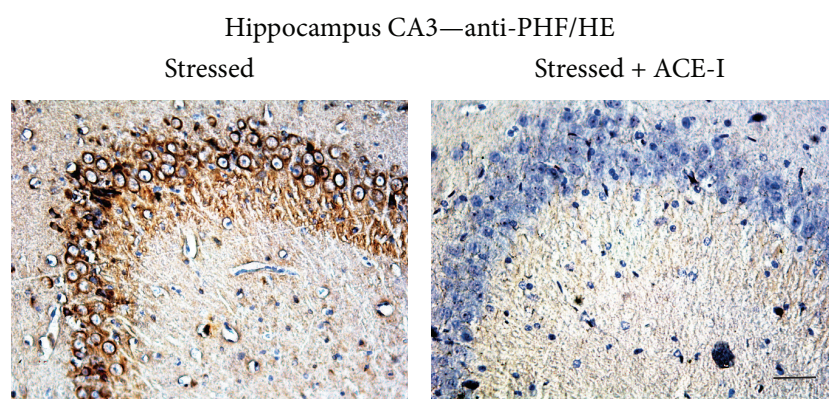

(a)

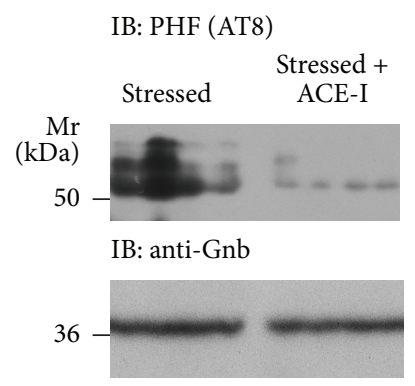

(b)

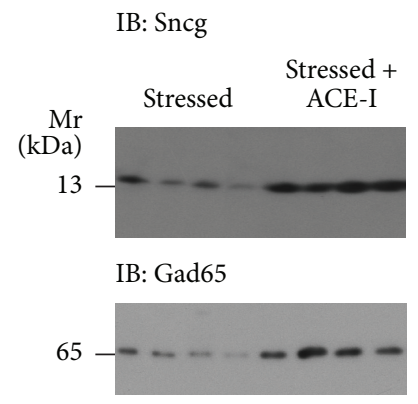

(c)

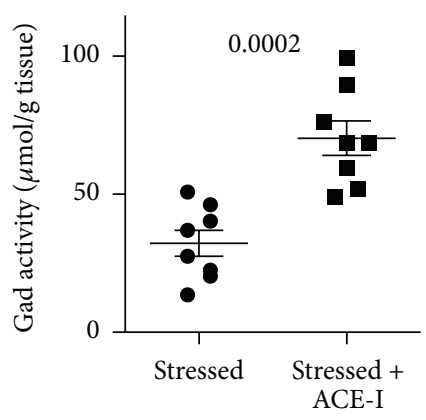

(d)

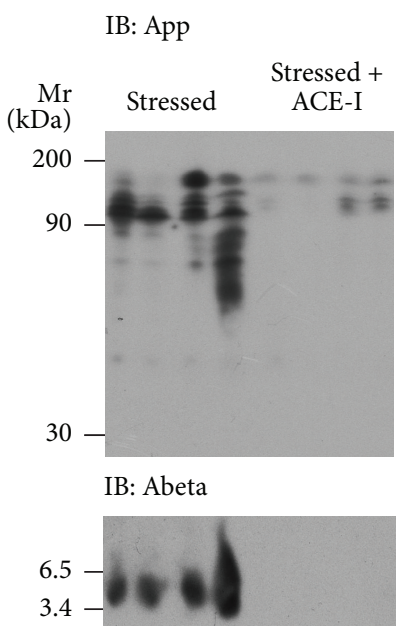

(e)

Figure 4: Treatment with the brain-penetrating ACE inhibitor captopril retarded signs of neurodegeneration induced by chronic mild stress. (a) Immunohistological detection of hyperphosphorylated tau protein with anti-PHF antibody in hippocampal CA3 neurons of a stressed rat without treatment (left panel) relative to a stressed rat treated with the ACE inhibitor captopril (Stressed + ACE-I) during the stress protocol (right panel); bar: $40 \mu \mathrm{m}$. Immunohistological experiments are representative of 4 rats/group. (b) Immunoblot detection of hyperphosphorylated tau protein (IB: PHF (AT8)) in hippocampal extracts of stressed rats without treatment relative to stressed rats treated with the ACE inhibitor captopril (Stressed + ACE-I) during the stress protocol ( $n=4 /$ group). (c) Immunoblot detection of Sncg (upper panel) and Gad65 (lower panel) in hippocampal extracts of stressed rats without treatment relative to stressed rats with ACE inhibitor (Stressed + ACE-I) treatment ( $n=4$ /group). (d) Hippocampal Gad activity of stressed rats without treatment relative to stressed rats treated with the $A C E$ inhibitor (Stressed + ACE-I) captopril ( $n=8$ /group; $P=0.0002)$. (e) Immunoblot detection of the amyloid beta (A4) precursor protein, App (IB: App; upper panel), and $\beta$-amyloid (IB: Abeta; lower panel) in hippocampal extracts of stressed rats without treatment relative to stressed rats treated with the ACE inhibitor captopril (Stressed + ACE-I) during the stress protocol ( $n=4 /$ group).

determined the hippocampal tau phosphorylation. Immunohistology analysis revealed a substantially decreased level of hyperphosphorylated tau protein in hippocampal CA3 neurons of a stressed rat with captopril treatment relative to that of a stressed control without $A C E$ inhibitor treatment (Figure 4(a)). Immunoblot detection of hyperphosphorylated tau confirmed immunohistology data and demonstrated the decreased hyperphosphorylated tau protein content in 
the hippocampus of stressed rats with captopril treatment relative to that of stressed rats without treatment (Figure 4(b)). Concomitantly, with the decreased tau phosphorylation, the stress-induced loss of synuclein-gamma (Sncg) was also retarded by $A C E$ inhibition with captopril (Figure 4(c), upper panel).

We next assessed the hippocampal Gad65 protein level and activity. Immunoblot analysis showed that captopril treatment counteracted the stress-induced decrease in hippocampal Gad65 protein (Figure 4(c), lower panel). The preserved Gad65 protein was accompanied by a significantly higher hippocampal Gad activity in stressed rats with captopril treatment compared to that in stressed rats without treatment (Figure 4(d)). Taken together, our data indicate that inhibition of $A C E$ with a brain-permeable $A C E$ inhibitor retarded the stress-induced tau hyperphosphorylation and counteracted the downregulation of Gad activity triggered by chronic mild stress.

Previous studies indicate that chronic mild stress enhances the generation of $\mathrm{A} \beta$ as a major neuropathological feature of $\mathrm{AD}[12]$ while $A C E$ inhibition decreases the hippocampal $\mathrm{A} \beta$ level, as documented in a genetic AD model [20]. The increased $\mathrm{A} \beta$ generation could be a consequence of the stressinduced increase in the steady-state level of the amyloid beta (A4) precursor protein (App) [32]. Complementary to those data, immunoblot analysis detected the high hippocampal App load in stressed rats without treatment whereas the hippocampal App content was substantially decreased in stressed rats with $A C E$ inhibitor treatment (Figure 4(e), upper panel). Concomitantly, immunoblot analysis revealed that the hippocampal $\mathrm{A} \beta$ level was also decreased in stressed rats with ACE inhibitor treatment compared to that in stressed rats without treatment (Figure 4(e), lower panel).

\section{Discussion}

4.1. Chronic Unpredictable Mild Stress Triggered Signs of Hippocampal Neurodegeneration. Our study applied the chronic unpredictable mild stress model, which imitates major features of sporadic AD such as tau hyperphosphorylation, enhanced $\mathrm{A} \beta$ generation, and other signs of dendritic and synaptic degeneration $[7,12]$. In agreement with those previous studies, we also found that chronic mild stress triggered signs of hippocampal neurodegeneration, which was documented by hippocampal tau hyperphosphorylation, decreased levels of major neuronal proteins such as Map2 and Sncg, and an increased level of A $\beta$-generating App. Concomitantly, with AD-related signs of neurodegeneration, glutamate excitotoxicity-enhancing features appeared such as decreased Gad65 protein level and enzyme activity and decreased hippocampal expression of the glutamateinhibitory NMDA receptor subtype, Grin3a (NR3A). The ensuing sensitization of glutamate excitotoxicity could contribute to stress-induced signs of neurodegeneration because glutamate excitotoxicity is a major factor, which contributes to neurodegeneration and tau hyperphosphorylation [29].
4.2. Chronic Mild Stress Induced Upregulation of Hippocampal Ace Protein and Activity. In search for additional stimuli, which could account for the development of stress-induced signs of neurodegeneration, our study detected a significantly increased hippocampal protein level of the angiotensin IIgenerating enzyme, Ace. Immunohistology analysis localized the upregulated Ace protein not only in brain vessels but also in neuronal cell bodies of the hippocampus. Concomitantly, an increased hippocampal Ace activity and angiotensin II peptide level were detected. The upregulation of neuronal Ace expression and protein level could be a direct consequence of the stress-induced activation of the hypothalamic pituitary adrenocortical (HPA) axis because several studies found that $A C E$ expression was triggered by glucocorticoids and glucocorticoid receptor activation [33-35].

4.3. ACE Inhibition Retarded Signs of Neurodegeneration Induced by Chronic Mild Stress. The upregulated Ace protein could be causally involved in signs of neurodegeneration triggered by chronic mild stress because treatment with the brain-penetrating $A C E$ inhibitor, captopril, retarded the development of signs of stress-induced neurodegeneration, that is, tau hyperphosphorylation, downregulation of neuronal proteins, decreased Gad enzyme activity, and increased hippocampal App level.

The mechanism underlying the neuroprotective activity of central $A C E$ inhibition is not entirely understood. Several studies indicated that the neuroprotective effect of central $A C E$ inhibition is largely due to a decrease in angiotensin II generation and subsequently blunted activation of the angiotensin II AT1 receptor, AT1R [20, 36-40]. Such conclusion is supported by data, which show the involvement of angiotensin II-AT1R in major neuropathological features such as tau hyperphosphorylation [37], enhanced glutamate release [38], and increased $A \beta$ formation [20, 39, 40]. In addition to direct neuroprotection, inhibition of the ACEAT1R axis could also counteract the stress response by blunting the release of ACTH [41]. In agreement with a role of the $A C E$-angiotensin II-AT1R axis in stress-induced neurodegeneration, we found that the hippocampal angiotensin II content was increased by stress, and hippocampal neurons with hyperphosphorylated tau protein and overt neurodegeneration were AT1R-positive.

In view of the stress-induced upregulation of the hippocampal Ace and the neuroprotective activity of ACE inhibition in the chronic mild stress model, our data support a causative role of $A C E$ in stress-induced neurodegeneration. Because the model reproduces major features of sporadic $\mathrm{AD}$ (i.e., tau hyperphosphorylation, $\mathrm{A} \beta$ generation, and glutamate excitotoxicity), our study could provide a rationale for the documented efficacy of centrally active $A C E$ inhibitors in several clinical studies, which showed retardation of $\mathrm{AD}$ related cognitive decline as a major sign of neurodegeneration [21-25]. In this context, the suggested repurposing of $A C E$ inhibitors for clinical conditions related to $\mathrm{AD}$ and other types of dementia [42] could be a potential option, which deserves further study. 


\section{Conclusion}

Inhibition of $A C E$ with the centrally acting captopril retarded the development of hippocampal tau phosphorylation, signs of neurodegeneration, and amyloid beta (A4) precursor protein (App) upregulation in rats subjected to chronic unpredictable mild stress, as a model, which reproduces major pathological features of sporadic $\mathrm{AD}$.

\section{Conflict of Interests}

The authors have no conflict of interests.

\section{Acknowledgment}

This study was supported in part by ETH Research Grant ETH-18 14-2.

\section{References}

[1] T. Scully, "Demography: to the limit," Nature, vol. 492, no. 7427, pp. S2-S3, 2012.

[2] A. Abbott, "Dementia: a problem for our age," Nature, vol. 475, no. 7355, pp. S2-S4, 2011.

[3] E. Marcello, F. Gardoni, and M. Di Luca, “Alzheimer's disease and modern lifestyle: what is the role of stress?" Journal of Neurochemistry, vol. 134, no. 5, pp. 795-798, 2015.

[4] P. Willner, A. Towell, D. Sampson, S. Sophokleous, and R. Muscat, "Reduction of sucrose preference by chronic unpredictable mild stress, and its restoration by a tricyclic antidepressant," Psychopharmacology, vol. 93, no. 3, pp. 358-364, 1987.

[5] M. Cuadrado-Tejedor, A. Ricobaraza, J. Del Río et al., "Chronic mild stress in mice promotes cognitive impairment and CDK5-dependent tau hyperphosphorylation," Behavioural Brain Research, vol. 220, no. 2, pp. 338-343, 2011.

[6] R. Alonso, G. Griebel, G. Pavone, J. Stemmelin, G. Le Fur, and P. Soubrié, "Blockade of $\mathrm{CRF}_{1}$ or $\mathrm{V}_{\mathrm{lb}}$ receptors reverses stress-induced suppression of neurogenesis in a mouse model of depression," Molecular Psychiatry, vol. 9, no. 3, pp. 278-286, 2004.

[7] H. Qiao, S. C. An, W. Ren, and X. M. Ma, "Progressive alterations of hippocampal CA3-CA1 synapses in an animal model of depression," Behavioural Brain Research, vol. 275, pp. 191-200, 2014.

[8] Y. A. El-faramawy, M. H. El-banouby, P. Sergeev, A. K. Mortagy, M. S. Amer, and A. M. Abdel-tawab, "Changes in glutamate decarboxylase enzyme activity and tau-protein phosphorylation in the hippocampus of old rats exposed to chronic mild stress: reversal with the neuronal nitric oxide synthase inhibitor 7-nitroindazole," Pharmacology Biochemistry and Behavior, vol. 91, no. 3, pp. 339-344, 2009.

[9] S. AbdAlla, H. Lother, A. El Missiry et al., "Angiotensin II AT2 receptor oligomers mediate G-protein dysfunction in an animal model of Alzheimer disease," Journal of Biological Chemistry, vol. 284, no. 10, pp. 6554-6565, 2009.

[10] M. Cuadrado-Tejedor, A. Ricobaraza, D. Frechilla, R. Franco, A. Pérez-Mediavilla, and A. Garcia-Osta, "Chronic mild stress accelerates the onset and progression of the Alzheimer's disease phenotype in TG2576 mice," Journal of Alzheimer's Disease, vol. 28, no. 3, pp. 567-578, 2012.
[11] M. Cuadrado-Tejedor and A. Garcia-Osta, "Chronic mild stress assay leading to early onset and propagation of Alzheimer's disease phenotype in mouse models," in Systems Biology of Alzheimer's Disease, vol. 1303 of Methods in Molecular Biology, pp. 241-246, Springer, New York, NY, USA, 2016.

[12] A. Briones, S. Gagno, E. Martisova et al., "Stress-induced anhedonia is associated with an increase in Alzheimer's diseaserelated markers," British Journal of Pharmacology, vol. 165, no. 4, pp. 897-907, 2012.

[13] R. S. Doody, R. G. Thomas, M. Farlow et al., "Phase 3 trials of solanezumab for mild-to-moderate alzheimer's disease," The New England Journal of Medicine, vol. 370, no. 4, pp. 311-321, 2014.

[14] S. Salloway, R. Sperling, N. C. Fox et al., "Two phase 3 trials of bapineuzumab in mild-to-moderate Alzheimer's disease," The New England Journal of Medicine, vol. 370, no. 4, pp. 322-333, 2014.

[15] E. Karran and J. Hardy, "Antiamyloid therapy for Alzheimer's disease-are we on the right road?" The New England Journal of Medicine, vol. 370, no. 4, pp. 377-378, 2014.

[16] M. L. Hemming and D. J. Selkoe, "Amyloid $\beta$-protein is degraded by cellular angiotensin-converting enzyme (ACE) and elevated by an ACE inhibitor," Journal of Biological Chemistry, vol. 280, no. 45, pp. 37644-37650, 2005.

[17] E. A. Eckman, S. K. Adams, F. J. Troendle et al., "Regulation of steady-state beta-amyloid levels in the brain by neprilysin and endothelin-converting enzyme but not angiotensin-converting enzyme," The Journal of Biological Chemistry, vol. 281, no. 41, pp. 30471-30478, 2006.

[18] K. E. Bernstein, Y. Koronyo, B. C. Salumbides et al., "Angiotensin-converting enzyme overexpression in myelomonocytes prevents Alzheimer's-like cognitive decline," The Journal of Clinical Investigation, vol. 124, no. 3, pp. 1000-1012, 2014.

[19] Y.-F. Dong, K. Kataoka, Y. Tokutomi et al., "Perindopril, a centrally active angiotensin-converting enzyme inhibitor, prevents cognitive impairment in mouse models of Alzheimer's disease," The FASEB Journal, vol. 25, no. 9, pp. 2911-2920, 2011.

[20] S. AbdAlla, A. Langer, X. Fu, and U. Quitterer, "ACE inhibition with captopril retards the development of signs of neurodegeneration in an animal model of Alzheimer's disease," International Journal of Molecular Sciences, vol. 14, no. 8, pp. 16917-16942, 2013.

[21] T. Ohrui, N. Tomita, T. Sato-Nakagawa et al., "Effects of brainpenetrating ACE inhibitors on Alzheimer disease progression," Neurology, vol. 63, no. 7, pp. 1324-1325, 2004.

[22] M. E. Soto, G. A. van Kan, F. Nourhashemi et al., "Angiotensinconverting enzyme inhibitors and Alzheimer's disease progression in older adults: results from the Réseau sur la Maladie d'Alzheimer Français cohort," Journal of the American Geriatrics Society, vol. 61, no. 9, pp. 1482-1488, 2013.

[23] Y. Gao, R. O’Caoimh, L. Healy et al., "Effects of centrally acting ACE inhibitors on the rate of cognitive decline in dementia," BMJ Open, vol. 3, no. 7, Article ID e002881, 2013.

[24] R. O’Caoimh, L. Healy, Y. Gao et al., "Effects of centrally acting angiotensin converting enzyme inhibitors on functional decline in patients with alzheimer's disease," Journal of Alzheimer's Disease, vol. 40, no. 3, pp. 595-603, 2014.

[25] F. F. de Oliveira, P. H. F. Bertolucci, E. S. Chen, and M. C. Smith, "Brain-penetrating angiotensin-converting enzyme inhibitors and cognitive change in patients with dementia due to Alzheimer's disease," Journal of Alzheimer's Disease, vol. 42, supplement 3, pp. S321-S324, 2014. 
[26] N. Mei, J. C. Fuscoe, E. K. Lobenhofer, and L. Guo, "Application of microarray-based analysis of gene expression in the field of toxicogenomics," Methods in Molecular Biology, vol. 597, pp. 227-241, 2010.

[27] L. Guo, E. K. Lobenhofer, C. Wang et al., "Rat toxicogenomic study reveals analytical consistency across microarray platforms," Nature Biotechnology, vol. 24, no. 9, pp. 1162-1169, 2006.

[28] J. Yan, X.-B. Sun, H.-Q. Wang et al., "Chronic restraint stress alters the expression and distribution of phosphorylated tau and MAP2 in cortex and hippocampus of rat brain," Brain Research, vol. 1347, pp. 132-141, 2010.

[29] P. Couratier, M. Lesort, P. Sindou, F. Esclaire, C. Yardin, and J. Hugon, "Modifications of neuronal phosphorylated $\tau$ immunoreactivity induced by NMDA toxicity," Molecular and Chemical Neuropathology, vol. 27, no. 3, pp. 259-273, 1996.

[30] J. H. Lee, Z. Z. Wei, D. Chen, X. Gu, L. Wei, and S. P. Yu, “A neuroprotective role of the NMDA receptor subunit GluN3A (NR3A) in ischemic stroke of the adult mouse," American Journal of Physiology-Cell Physiology, vol. 308, no. 7, pp. C570C577, 2015.

[31] H. Wang, H. Yan, S. Zhang, X. Wei, J. Zheng, and J. Li, "The GluN3A subunit exerts a neuroprotective effect in brain ischemia and the hypoxia process," American Society for Neurochemistry Neuro, vol. 5, no. 4, pp. 231-242, 2013.

[32] K. N. Green, L. M. Billings, B. Roozendaal, J. L. McGaugh, and F. M. LaFerla, "Glucocorticoids increase amyloid- $\beta$ and tau pathology in a mouse model of Alzheimer's disease," Journal of Neuroscience, vol. 26, no. 35, pp. 9047-9056, 2006.

[33] M. L. M. Barreto-Chaves, I. Anèas, and J. E. Krieger, "Glucocorticoid regulation of angiotensin-converting enzyme in primary culture of adult cardiac fibroblasts," American Journal of Physiology-Regulatory Integrative and Comparative Physiology, vol. 280, no. 1, pp. R25-R32, 2001.

[34] Y. Dasarathy, J. J. Lanzillo, and B. L. Fanburg, "Stimulation of bovine pulmonary artery endothelial cell ACE by dexamethasone: involvement of steroid receptors," American Journal of Physiology-Lung Cellular and Molecular Physiology, vol. 263, no. 6, pp. L645-L649, 1992.

[35] R. S. Fishel, S. Eisenberg, S. Y. Shai, R. A. Redden, K. E. Bernstein, and B. C. Berk, "Glucocorticoids induce angiotensinconverting enzyme expression in vascular smoth muscle," Hypertension, vol. 25, no. 3, pp. 343-349, 1995.

[36] S. Villapol and J. M. Saavedra, "Neuroprotective effects of angiotensin receptor blockers," American Journal of Hypertension, vol. 28, no. 3, pp. 289-299, 2015.

[37] M. Tian, D. Zhu, W. Xie, and J. Shi, "Central angiotensin II-induced Alzheimer-like tau phosphorylation in normal rat brains," FEBS Letters, vol. 586, no. 20, pp. 3737-3745, 2012.

[38] Y.-M. Kang, D.-M. Zhang, X.-J. Yu et al., "Chronic infusion of enalaprilat into hypothalamic paraventricular nucleus attenuates angiotensin II-induced hypertension and cardiac hypertrophy by restoring neutrotransmitters and cytokines," Toxicology and Applied Pharmacology, vol. 274, no. 3, pp. 436444, 2014.

[39] D. Zhu, J. Shi, Y. Zhang et al., "Central angiotensin II stimulation promotes $\beta$ amyloid production in Sprague Dawley rats," PLoS ONE, vol. 6, no. 1, Article ID e16037, 2011.

[40] J. Liu, S. Liu, Y. Matsumoto et al., "Angiotensin type la receptor deficiency decreases amyloid $\beta$-protein generation and ameliorates brain amyloid pathology," Scientific Reports, vol. 5, article 12059, 2015.
[41] N. Glorioso, P. Dessifulgheri, S. Alagna et al., "Angiotensin converting enzyme inhibition reduces ACTH release due to hypoglycaemia," Clinical and Experimental Hypertension A, Theory and Practic, vol. 9, no. 2-3, pp. 665-670, 1987.

[42] X.-Z. Zhang, Y. Quan, and G.-Y. Tang, "Medical geneticsbased drug repurposing for Alzheimer's disease," Brain Research Bulletin, vol. 110, pp. 26-29, 2015. 


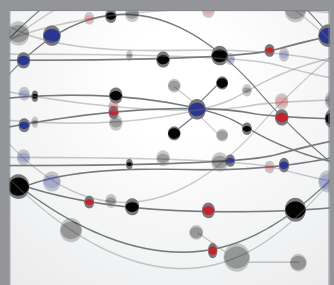

The Scientific World Journal
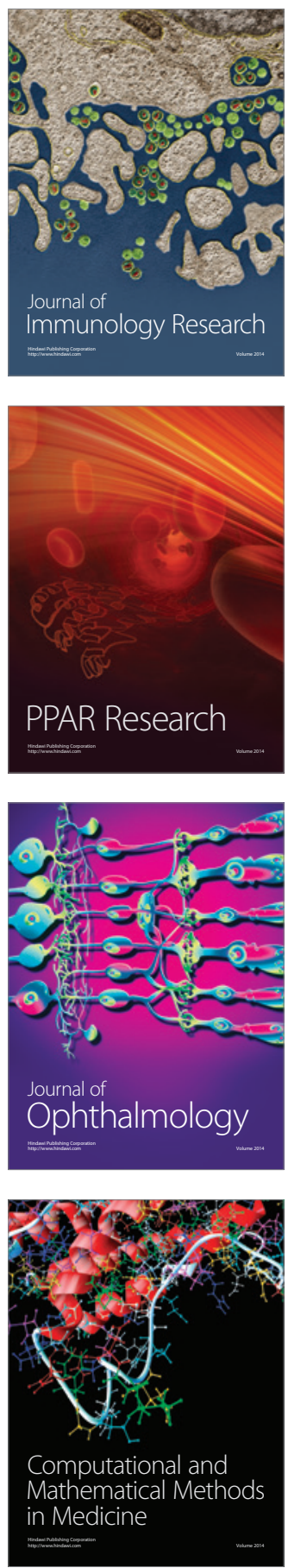

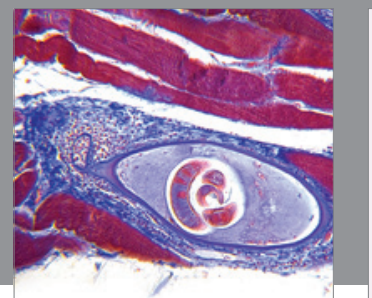

Gastroenterology

Research and Practice
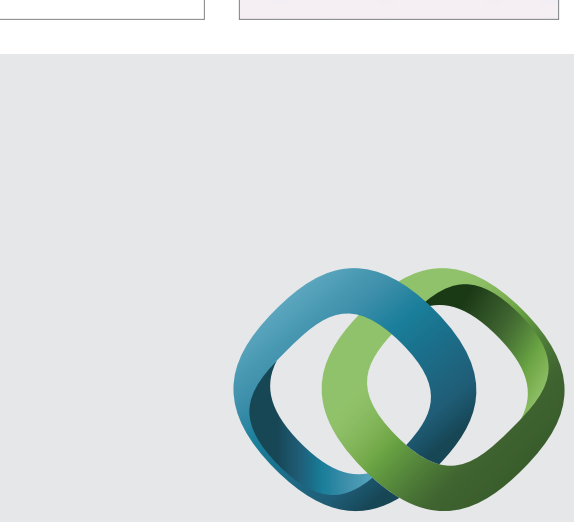

\section{Hindawi}

Submit your manuscripts at

http://www.hindawi.com
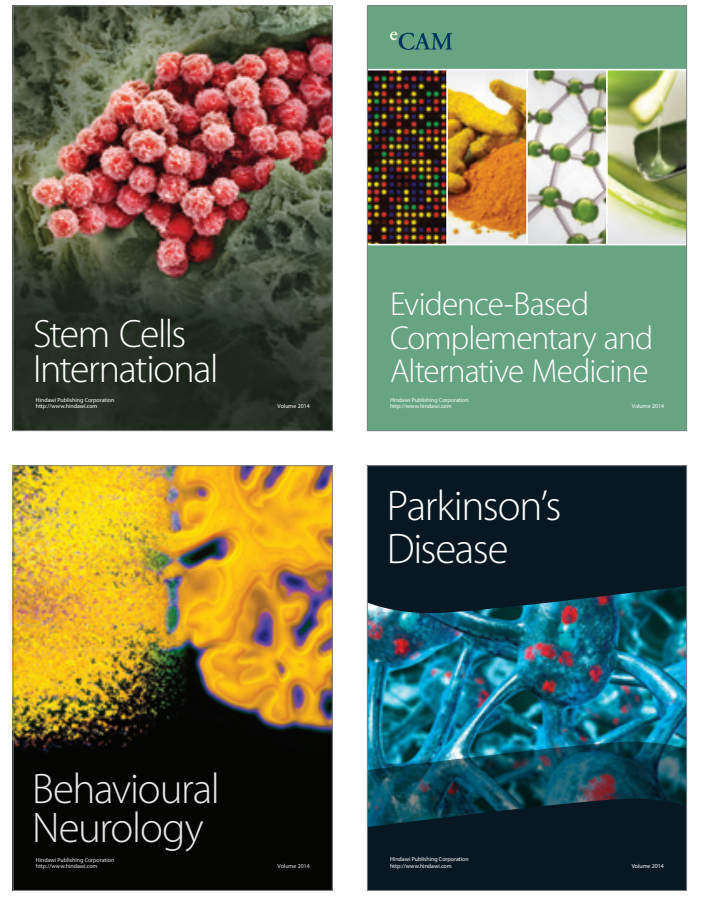
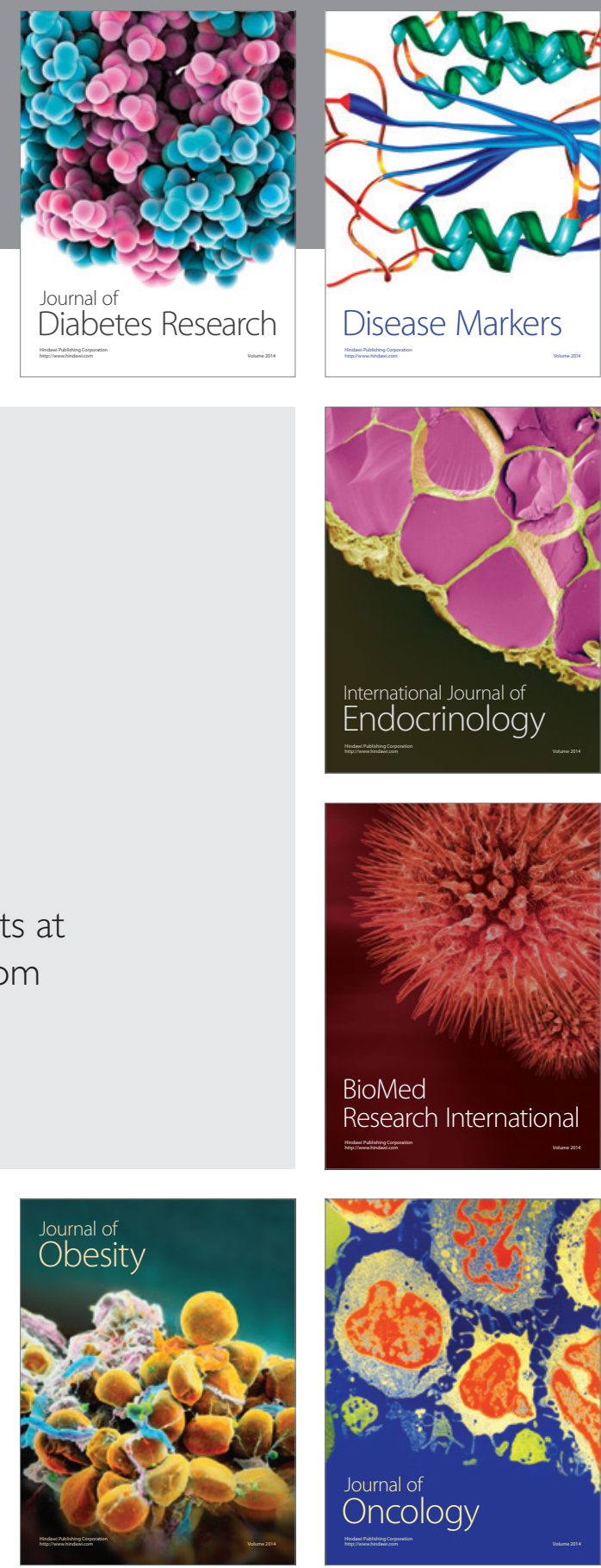

Disease Markers
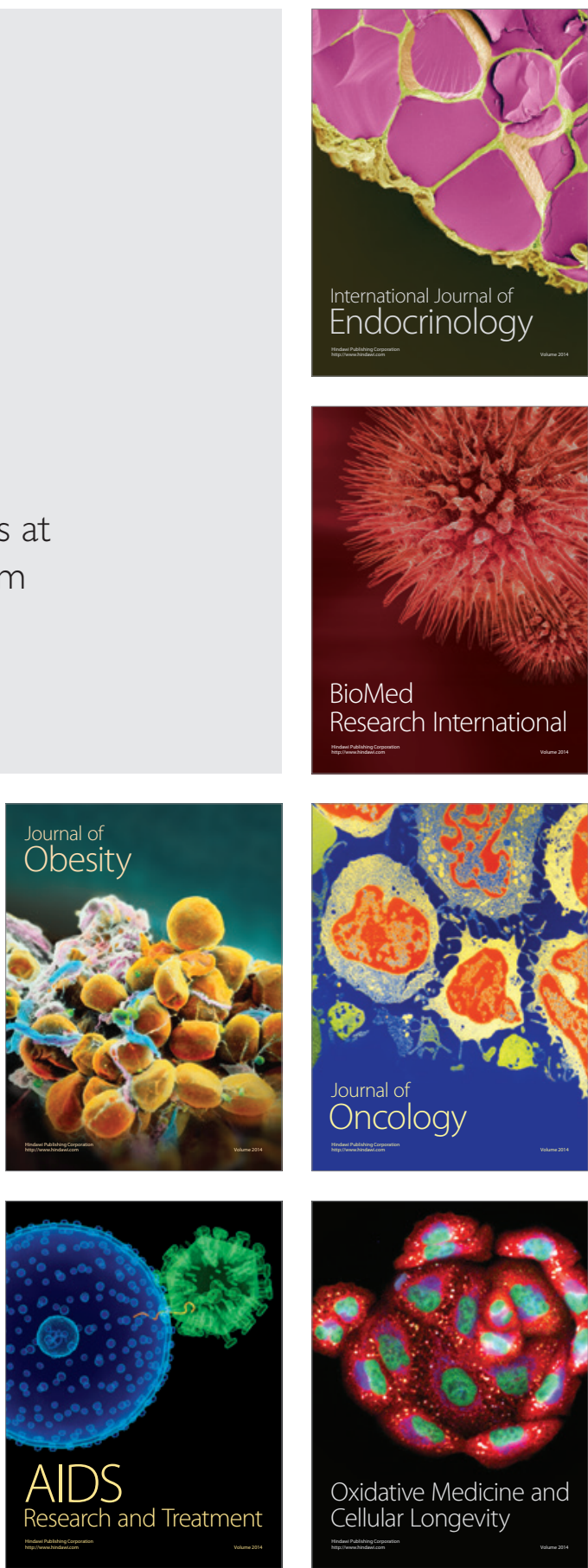